\title{
A Reasoned Approach to the Integration of Design and Fabrication Technologies in Architecture Education \\ Giancarlo Di Marco
}

Centro de Diseño, Cine y Televisión, Department of Industrial Design, Mexico City, Mexico

\section{Abstract}

Integrating industrial technologies within education curricula, as well as creating fabrication laboratories, has become inevitable for Architecture Departments: digital design tools (eg: CG and CAD software) and digital fabrication tools (eg: cutting, printing, and milling technologies) have become standard assets and almost every University has a digital fabrication lab, or at least a 3D printer, or a laser cutting machine.

The education system itself is striving to find new pedagogic methods to best involve students in the learning process: learning-by-doing, project-based learning, gamification, "tailored" curricula, etc. All of these methods require a simplification of the

Corresponding Author: Giancarlo Di Marco

gdimarco@centro.edu.mx

Received: 15 March 2019 Accepted: 25 May 2019 Published: 20 November 2019

Publishing services provided by Knowledge $\mathrm{E}$

(c) Giancarlo Di Marco. This article is distributed under the terms of the

Attribution License, which permits unrestricted use and redistribution provided that the original author and source are credited.

Selection and Peer-review under the responsibility of the Architecture across Boundaries Conference Committee. basic theoretical set of knowledge which is fundamental in any profession, especially in Architecture. Moreover, the first outcomes of applied learning-by-doing programs show that such programs are as time-demanding as traditional programs [1]. On the other hand, the use of advanced tools such as parametric/generative software and prototyping/fabrication machines demands a high degree of interdisciplinary preparation, always crossing the boundaries of the specific field of study.

Therefore, the integration of design and fabrication technologies in Architecture Education is not a simple process and it requires a redesign of the whole curriculum as well as the creation of an educational transition system from secondary schools to university.

Switching from standard architectural design processes to advanced digital design and fabrication enhanced processes is possible by learning how to code.

Keywords: Parametric Design, Digital Fabrication, Machine Learning, Coding, Education, STEM, Transdisciplinary, Curriculum Theory

\section{Introduction}

Considering the recent developments on artificial intelligence and machine learning, a pattern emerges indicating how there is or there should be one common approach [2] to all (design) activities and this approach is based on coding.

As for Architectural Design, coding allows performing optimisation and form-finding operations in an infinite set of environmental and functional variables [3].

Coding is also the core of current design techniques such as parametric/generative/ computational design as well as digital fabrication. 
With coding being the powerful ideal tool for future Architects, some fundamental steps towards the integration of design and fabrication technologies in education have already been taken.

We limit the analysis to those technologies that are already used in Architecture: CAD / BIM / CG software or "design technologies"; CNC machines or "fabrication technologies".

CAD / BIM / CG software is already part of the architectural design workflow.

The first CAD software dates back to the early 1960s [4] (Figure 1). After 60 years, design technologies offer good usability and are widely used both in academia and in professional practice.

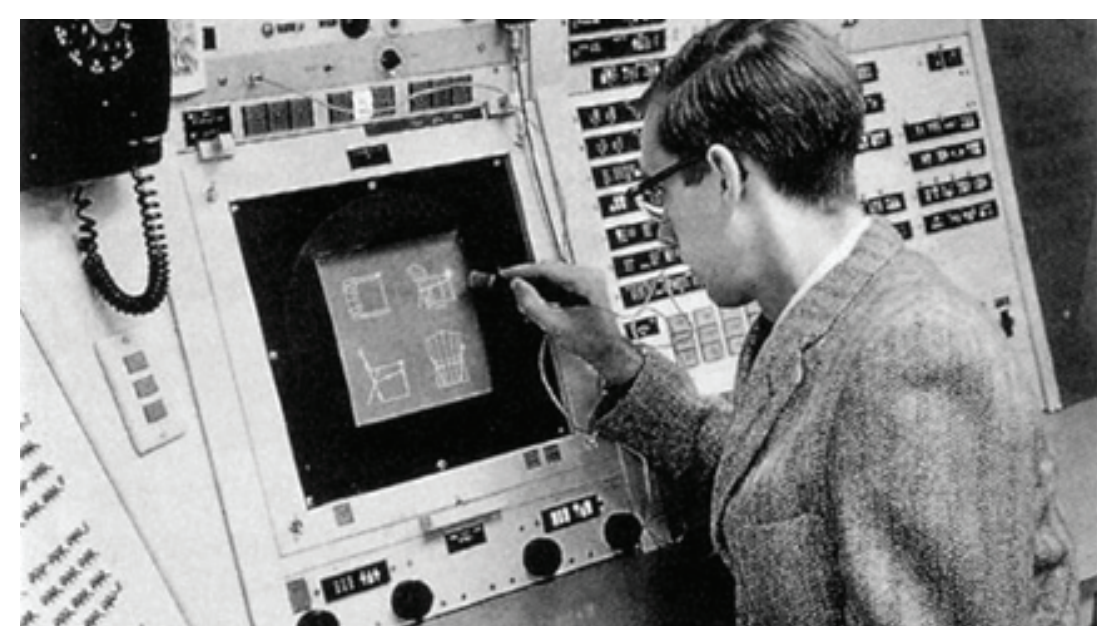

Figure 1: Ivan Sutherland's SKETCHPAD, MIT.

Today, we're on the verge of the integration of CNC machines (fabrication technologies) in Architecture education.

The first 3D printer was created in the early 1980s and in 2009 the patent for FDM 3D printing technology expired allowing 3D printers to become a commercial product. Still at that time, after 30 years, 3D printing was still used for non-functional prototyping only or, in a few specific cases as part of production processes. After 40 years since 3D printing was invented, the first architectural-scale applications are starting to appear: small 3D printed houses (Figure 2), concrete or metallic footbridges, etc.

The impact that new technologies have on consolidated human activities and industrial processes can be measured over the decades, nevertheless, in the last ten years, design and fabrication technologies have started spreading in the professional and industrial environment at a very fast rate even if their average use consists in making traditional processes more efficient or less expensive. 


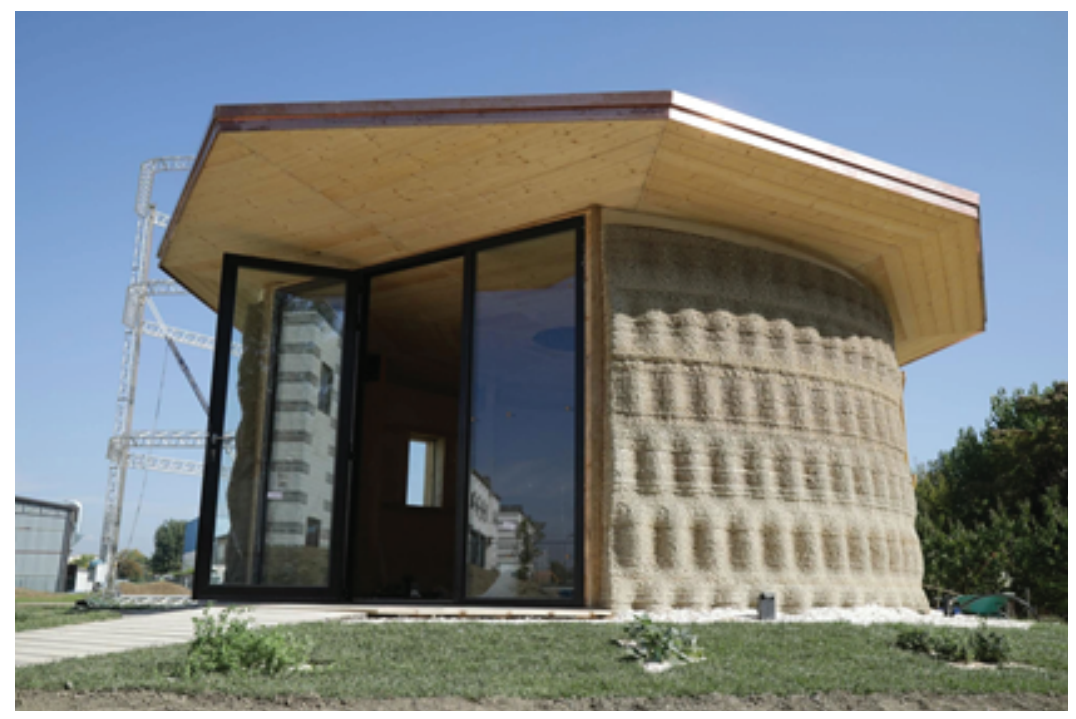

Figure 2: "Gaia", a 3D printed mud house, WASP.

At the same time, the academia understands the importance of this phenomenon and keeps striving to integrate such technologies to fill the gap with the industry but with outcomes that are still far from being considered at high quality.

\section{Purpose}

The purpose of this study is to propose a toolkit for Universities, to help them exploring and defining the best strategy for integrating design and fabrication technologies within architecture education.

\section{Methodology}

This paper presents the first outcome of a four years study conducted on:

- Eight generations of degree and postgraduate students in a design university in Mexico City, with about 400 students involved.

- A network of thousands of professionals/students from all over the world.

- 23 companies from Europe, the USA, and Latin America.

Additional information has been collected by speaking with professors, students and professionals during lectures and masterclasses in various universities, as well as during consulting activities with Architecture, Engineering, and Design firms.

Instruments used: 
- Direct interrogation of participants.

- Tests aimed at evaluating knowledge and understanding.

- Practical exercises.

- Anonymous surveys.

- Analysis of Faculties curricula.

The results also include findings from previous research by the author on the relation between "digital natives" [5, 6] (typically the Generation $Y$ or Millennials students) and education.

\section{Findings}

There is a prevailing confusion on what is necessary to properly approach complex subjects like design and fabrication. Because such technologies are directly connected to $3 \mathrm{D}$ modelling it is a common mistake to think that CAD, BIM or CG courses are fundamental. Therefore, the standard curricula do not include subjects for acquiring basic useful knowledge, or they don't deepen the subjects enough to allow a real and seamless integration of modern technologies.

Coding subjects are seldom present and appear mostly as parametric design (visual coding) courses in the last semesters of the program. The most used programming language seems to be Processing, followed by Python. Despite the fact that Processing is the core of interactive design (eg: Arduino) and a big part of the maker movement is based on this particular programming language, Processing itself is not the best choice for design and fabrication processes while Python is.

Cutting-edge fabrication technologies are present in many university labs but they are normally under-used. It takes years to figure out how such technologies can be efficiently used in an educational environment and to adapt the curricula for their integration.

In many cases, fabrication labs become independent entities or spin-offs so it is possible to amortize the cost of the machines, while in some other cases they incorporate a business incubator where startup companies working with design and fabrication processes can rent and share expensive technologies without having to invest.

Students preparation is not on average up to a level of academic content, with serious knowledge gaps and poor ability to inductive-deductive reasoning.

Students claim to possess specific knowledge but: $90 \%$ have problems explaining design-relevant concepts such as the difference between parametric and organic, 
or curved and organic; 50\% cannot give an exact definition of "circle"; $50 \%$ doesn't know how to use analog measuring instruments; $60 \div 70 \%$ doesn't know derivatives and geometric continuity.

As for Architecture Schools, in addition to all that has already been described, most of the examined programs do not include coding subjects, even if a change is recently arising with the introduction of parametric design (eg: Grasshopper for Rhinoceros). It seems to be a common belief that such tools are easier to teach and learn towards the end of the program, between 6th and 8th semester. After years of using traditional design methods, students are asked to suddenly switch to a

procedural-based one: one-three semesters are absolutely insufficient for filling the gap that leads to advanced design and fabrication applications [7]

On the other hand, the transition from simple to complex geometry via NURBS surfacing and parametric design generates a big impact on students' minds: organic shapes and patterns become relatively easy to draw so students tend to design complex shapes just because now they can! This is one example of a technology-driven design.

Fabrication labs started to appear only recently in Architecture Schools and they are mostly equipped with 3D printers, laser cutters and eventually 3 to 4 axes CNC mills. The main purpose of these labs is to create architectural models for students. For this reason, students don't need to have specific knowledge of fabrication technologies while machines can be operated by any technician with sufficient technical knowledge, most likely with non-existent architectural culture.

Technicians are also difficult to find since new fabrication technologies are quite expensive and are present mostly inside production plants: once a technician acquires such a specific knowledge he/she becomes precious for the employer. To make a comparison, these modern technology professionals are the equivalent of the model maker, one of the most paid professionals in the product design industry.

The majority of Architecture students don't know any programming language; those who know at least one design/fabrication-relevant programming language have learned it outside of the University or in workshops. The good news is that the few who know a programming language have studied Python and/or $\mathrm{C}++$, which are useful for design and fabrication.

In any case, almost all the students have no idea of what is machine learning and how it could affect the practice of Architecture. 
Considered as a pioneer in (architectural) design, the MIT BS in Architecture program includes subjects like Design Computing, Advanced Design Projects in Digital Fabrication, Advanced Visualization: Architecture in Motion Graphics, as well as a Restricted Electives "Computation" section at the Senior Year with three subjects: Design Scripting, Visual Computing 1, Visual Computing 2.

\subsection{Survey on Digital Fabrication and University}

A survey conducted on a selected sample of students and professionals led to the following conclusions (Table 1).

\section{Practical Implications}

According to the findings, a lot of work must be done in order to properly introduce our students to digital design and fabrication.

Considering subjects like parametric or computational design that are already present in some Architecture programs, they require a specific skill set that includes at least coding, geometry, differential geometry, and vector geometry. The same skill set is fundamental for fabrication technologies.

The implementation of digital fabrication laboratories inside Architecture Schools risks being unsuccessful if the program is not adjusted accordingly.

\section{Value}

There is no evidence of a reasoned approach to the integration of digital design and fabrication in academic programs, thus integration is often motivated only by the vision of the administrative bodies or by the academic council, driven by a strong intuition about the importance and value of the use of new technologies for improving academic and professional practice, and, at the same time, with little clarity about the goals and the method to perform such integration.

The value of this work is to underline the critical aspects of integrating design and manufacturing technologies into educational programs and to provide a draft for efficient planning and integration strategy. This can be useful for Architecture Schools and for any academic institution or professional entity. 
TABLE 1: Results from the survey on Digital Fabrication and University

\begin{tabular}{|c|c|}
\hline \multicolumn{2}{|r|}{ Studies } \\
\hline $100 \%$ & Have access to a computer lab at the University. \\
\hline $70 \%$ & Have access to a fabrication lab at the University. \\
\hline$>70 \%$ & Attended at least one Geometry course at University. \\
\hline$<50 \%$ & $\begin{array}{l}\text { Attended at least one Mathematical Analysis course at University. Considering } \\
\text { professionals were involved in this survey, we have reason to believe that this } \\
\text { percentage could be even lower after studying actual curricula from several } \\
\text { Architecture undergraduate programs. }\end{array}$ \\
\hline$<40 \%$ & Attended a coding course at University. \\
\hline$<15 \%$ & Attended a propaedeutic course before entering the University. \\
\hline $0 \%$ & $\begin{array}{l}\text { Attended a propaedeutic course relevant for a technologically enhanced } \\
\text { education program. }\end{array}$ \\
\hline \multicolumn{2}{|r|}{ Coding } \\
\hline $65 \%$ & Knows at least one programming language. \\
\hline$>45 \%$ & Knows Processing. \\
\hline$<30 \%$ & Knows Python. \\
\hline$<40 \%$ & $\begin{array}{l}\text { Knows something about } \mathrm{Al} \text { and machine learning. We have reason to believe this } \\
\text { percentage could be lower: after interviewing a sub- sample of } 100 \text { persons we } \\
\text { detected a lack of meaningful knowledge of the subject, especially from a } \\
\text { practical or technical point of view. }\end{array}$ \\
\hline \multicolumn{2}{|r|}{ Design } \\
\hline $80 \%$ & Has a good knowledge of digital design tools and 3D modeling. \\
\hline$>90 \%$ & Uses Rhinoceros as primary 3D modeling software. \\
\hline $20 \%$ & Uses Autodesk Revit Architecture (BIM). \\
\hline $33 \%$ & Has poor freehand drawing abilities. \\
\hline $20 \%$ & Has adequate Geometry knowledge for design and fabrication enhanced courses. \\
\hline$<40 \%$ & Knows first and second derivatives. \\
\hline$<40 \%$ & Knows the meaning of geometric continuity. \\
\hline \multicolumn{2}{|r|}{ Fabrication } \\
\hline$<30 \%$ & Owns a 3D printer. \\
\hline$>75 \%$ & Has used a 3D printer. \\
\hline $87 \%$ & Knows what is a CNC milling machine. \\
\hline $70 \%$ & Knows how a 3D scanner works. \\
\hline $50 \%$ & Knows what is G-code. \\
\hline $60 \%$ & Has been in a FabLab. \\
\hline \multicolumn{2}{|r|}{ Wishful Thinking } \\
\hline $95 \%$ & Thinks fabrication training is useful. \\
\hline
\end{tabular}




\section{Conclusion}

Considering the latest evolutions of CAD, BIM and digital fabrication, as well as the underlying code- based approach that all of these technologies share, I believe that the most efficient path towards a fruitful and seamless integration of design and fabrication technologies in Architecture Education is based on coding.

Universities that want to use digital design and fabrication with the goal of preparing new professionals capable of pushing the use of such technologies beyond the actual barrier of making traditional processes more efficient must make a radical change in their programs by introducing coding as an instrument for:

- The development of procedural thinking.

- Computational CAD and BIM applications.

- Managing and customizing digital fabrication processes and applications.

Below are the specific conclusions for the various objectives set.

\subsection{Self-Assessment}

Analysis of the academic external and internal environment.

This is the first draft of a survey that is meant for understanding or clarifying the academic goals related to the integration of design and fabrication technologies in education.

- External environment - This might be important or not, depending on the target of the University: international universities that prepare new professionals for the global market might consider the building industry at the global scale; on the other hand, universities with a smaller area of influence should focus on the local building industry characteristics.

- Is the building industry:

* Aware of advanced design/fabrication processes?

* Ready for advanced design/fabrication processes?

* Educated about advanced design/fabrication processes?

* Using advanced design/fabrication processes?

- Academic environment - Buying new technologies just because there is the vision and the budget is often the reason for poor integration outcomes. Most of 
the cutting edge technologies installed in university fabrication laboratories are under-used.

- What should the goal of design/fabrication technologies integration be:

* Prepare students to work inside existing technology-aware processes?

*Validate actual technological applications and results in a controlled environment?

* Investigate new uses for design/fabrication technologies?

- Does the curriculum contain all the necessary subjects for developing the right design/fabrication skill set?

- Does the staff (professors and technicians) possess all the necessary skills? If no:

${ }^{*}$ Is it possible to acquire such skills with training?

* Is additional specialized staff needed?

${ }^{*}$ Is the recruiter capable of recognizing and evaluating new staff members skills?

* Is an external consultant needed for recruiting specialized staff?

* Is the recruiter aware of how much a design/fabrication specialized employee might cost?

- Is the recruiter aware of all the adjustments that must be done before bringing a fabrication technology in the laboratory (dedicated electrical systems, disposal plants, safety, ventilation systems, sound insulation, vibration isolation, etc.)?

\subsection{Propaedeutic courses}

A strong understanding of Geometry, Mathematical Analysis, Differential Geometry, and Vector Geometry is necessary in order to work with design and fabrication technologies while new students generations present a significant knowledge gap, especially referred to these subjects.

This gap should be filled with a transition strategy from secondary school to University based on propaedeutic courses and/or an Associate of Arts (AA) transfer degree (two years of prerequisite courses).

Some visual computing tools allow the study of the aforementioned subjects in a more intuitive way and present the plus of making students familiarize with a computational design environment and with complex geometry. 


\subsection{Design technologies}

As already stated, design technologies are part of the standard architectural workflow and therefore they are easy to integrate properly in the Architecture curriculum with workshops and continuing education courses on 3D modeling, parametric design, BIM.

There are design technologies that best relate to fabrication processes, so it would be better to focus on software that can directly interact with fabrication.

Modern CAD tools allow the design of complex mathematical geometry (NURBS) [8] and therefore some notions about differential geometry are necessary.

Serial Architecture where modularity and standardization are mandatory, and Tectonic Architecture

[9] where unique organic shapes are integrated with the environment, are respectively associated with BIM and AAD [10] tools. The border between BIM and CAD is no longer rigidly defined thanks to coding: parametric/computational design is already a fundamental part of both software categories and skilled computational designers no longer use the main software but prefer to work directly with the computational plugins.

\subsection{Fabrication technologies}

Integrating fabrication technologies in Architecture Education is extremely difficult, mainly because of two factors:

- Some technologies are still unstable and/or unreliable (eg: 3D printing).

- Some technologies require a very specific set of knowledge with almost no connection with the curriculum of an Architect (eg: CNC milling).

Cut technologies (laser, plasma, water-jet) are easy to use but their applications in an architectural design and fabrication workflow require perfect knowledge of geometry, 3D modeling, and coding.

From a financial point of view, the "FabLab + Business Incubator" business model is possibly the best option for integrating design and fabrication technologies in the academic environment.

- Machines cost is amortized by renting machine time and office spaces.

- Incubated startup companies working with design and fabrication processes build a creative environment with fresh ideas on machines applications. 
- The innovative startup companies are the perfect connection between the academic world and the industry.

\subsection{Coding}

Cutting-edge trends such as computational design and machine learning, make it necessary to add coding to the education of the new Architect, focusing on those programming languages that best relate to design and fabrication technologies. At the beginning of 2019, Python scripting is probably the best option. Learning to code with a programming language like Python cannot be achieved with one single propaedeutic course, it rather requires a 1-year study possibly during the first year of the program.

\section{Conflict of Interest}

The author has no conflict of interest to declare.

\section{References}

[1] Nemorin, S. (2017). The frustrations of digital fabrication: an auto/ethnographic exploration of "3D Making" in school. International Journal of Technology \& Design Education, 27(4), 517-535.

[2] Domingos, P. (2018). The Master Algorithm: How the Quest for the Ultimate Learning Machine Will Remake Our World. The United States: Basic Books.

[3] Derix, C., and Jagannath, P. (2014). Near Futures: Associative Archetypes. Architectural Design, 84(5), 130-135.

[4] Sutherland, I. E. (1963). SKETCHPAD, A man-machine graphical communication system. Ph.D. dissertation/master's thesis, Massachusetts Institute of Technology.

[5] Pew Research Center. (March 2014). Millennials in Adulthood: Detached from Institutions, Networked with Friends.

[6] Junco, R. and Mastrodicasa, J. (2007). Connecting to the net generation: What higher education professionals need to know about today's students. Washington, DC, NASPA.

[7] Dunn, N. (2012). Digital Fabrication in Architecture. England, Laurence King Publishing Ltd.

[8] Di Marco, G. (2018). Simplified Complexity - Method for advanced NURBS modeling with Rhinoceros. Italy: Le Penseur. 
[9] Schumacher, P. (2017). Tectonism in Architecture, Design and Fashion: Innovations in Digital Fabrication as Stylistic Drivers. Architectural Design, 87(6), 106-113.

[10] Tedeschi, A. (2014). AAD_Algorithms-Aided Design - Parametric strategies using Grasshopper. Italy: Le Penseur. 